\title{
Consciousness! Look at the Light
}

\author{
Alemdar $\mathbf{E}^{*}$ \\ Goce Delcev University Medicine Faculty, North Macedonia, Turkey \\ *Corresponding author: Eda Alemdar, Goce Delcev University Medicine Faculty North Macedonia, Turkey
}

\begin{abstract}
ARTICLE INFO
Received: 幽 February 06, 2020

Published: 㓞 February 12, 2020

Citation: Alemdar E. Consciousness! Look at the Light. Biomed J Sci \& Tech Res 25(4)2020. BJSTR. MS.ID.004224.

\section{ABSTRACT}

According to the Big Bang theory, there is light in the first formation of the universe. This light exists in the entire universe. Light, which is the source of life for living things, is also the source of life for the universe. All living things with or without direct light benefit from light. All brain-centered functions of human organs are realized by light. In these functions, light is the main factor; because it is light that drives the brain. In fact, even though the brain is separate from the body, the functions of the brain can take place throughout the human body. There are multiple variants of light. Among many others, cosmic dark light is the source of known and dominant light throughout the universe.
\end{abstract}

Keywords: Consciousness; Light; Cosmic dark; Human
Abbreviations: QIS: Quantum Information Science; QDs: Quantum Dots; LED: Light-Emitting Diode

\section{Introduction}

\section{Light}

There are two kinds of thesis about the first formation of the universe. The Big Bang, the most powerful of the two concepts, is a cosmological model that explains the beginning and evolution of the universe. This thesis successfully describes many physical phenomena, including cosmic radiation, the large-scale structure of the universe, Hubble's law, and the abundance of light elements in the universe. In experiments with large particle accelerators, many details about the Big Bang model can be confirmed by creating conditions in the early universe. But since such high energy intensities immediately after the Big Bang have not been reached, the details of the first moments of the universe are still controversial. The most significant factor in the formation of the big explosion is light. Thus, in the first formation of the universe, light is a determining and shaping factor [1].

According to this widely held well-accepted theory, the stars were formed by cold hydrogen gas covering the universe after the Big Bang. Although they probably had huge magnitudes and incredible brightness, their lifespan was short. When they exploded, they spread the light and the chemical elements that made life possible in the universe. Accordingly, light is the most important factor in the formation of the universe. As a matter of fact, studies on the first formation, support this argument [2]. Meanwhile, according to sacred books light dominates the universe [3]. According to philosophers there is a soul at the core of the universe; and that soul is the light. The revival of all living things depends on the light. Indeed, the legal injunctions from the philosophers indicated alRuh to be extremely light. For example, according to Plato, the soul is like an eye; when the soul faces the light of truth and entity, it perceives and understands [4].

Light is also considered as the most effective factor in quantum theory, probably the most interesting theory of today [5]. As a matter of fact, a team of researchers from Northwestern University documented the role that chemistry will play for the first time in the next generation of computers and communications. By applying their expertise in the field of Quantum Information Science (QIS), they discovered how quantum information can be carried through nano-scale quantum irradiation (a subject of the QIS field). Thus, by generating entangled electrons through photochemistry that interact with a third electron in an organic radical, it can transfer information from one end of the molecule to the other by electron transfer and ensure that it is transported without compromising or altering the transmitted information. Wasielewski's Group is able to irradiate information across a molecule using an electron transfer mechanism that has never been achieved before [6]. This is light. Thus, quantum irradiation can be carried out between quantum systems of a different nature, just like between light and matter [7]. In terms of realizing a quantum theory of light, the photon-photon interactions required to realize quantum logic can be mediated by 
light-matter interactions in systems such as Quantum Dots (QDs) and color centers as the power of light leads to quantum fluctuations [8]. This shows that there is a serious connection between light and quantum.

\section{Light is Consciousness}

Light is a very powerful and sensitive tool that enables us to control the shape and create new stages of matter [9]. Light is the most important factor in the formation of human life. Moreover, light also has a significant impact on positive or negative developments in human health [10]. The brain is a remarkably complex system with activity patterns at a critical point between order and chaos, integrating inputs from different modalities into a unified experience in the world. Brain functions change over time and have been found to differ between the states of consciousness in both anesthetized non-human primates and in patients with impaired consciousness. The interaction of integration to support brain entropy and consciousness can occur temporally as well as spatially [10]. Although the relationship between brain and consciousness is not clear, the brain is accepted as the most important factor in the formation and development of humans and human products. Along this line, claims that consciousness takes place in the brain, gains weight. However, this approach doesn't provide all answers regarding consciousness.

Natural light is essential for securing our biological clocks and circadian rhythm [11]. This leads to physiological and behavioral time coordination [12]. Therefore, human consciousness has a direct connection with light; and it is light that creates consciousness. By seeing the light, the act of seeing takes place. Through light, the brain performs the functions of thinking, reasoning, processing, integrating, and interpreting all the information that it has. Thru such activities, light activates memory. Light is also a factor that activates human organs. The starting point of living things is light. The source of vision for living things is in general the eye, as the primary function of the eye is to interact with light, "When a person comes to the world, they opens their eyes, and so receive light. A person closes their eyes when they die, and so the light ends." This means that, instead of the eye, it is the light that enables human beings to see. Because to see, the eye is not actually mandatory. Babies also interact with light while in the womb [13]. In a newborn baby, the visual act occurs later. However, the newborn baby can carry out and perceive body functions to a great extent before being able to see with the eyes. This indicates that light has no direct connection to the eye. Because the eye performs the act of seeing in general, the eye is not necessary for recognition, understanding, perception, and realization. As a matter of fact, people can design without seeing.

Designing something that is unseen in the mind is the function of consciousness, not the eye. In this respect, consciousness has no mandatory connection with vision. Therefore, the act of vision, not the eye, provides consciousness. The eye activates the body system just by sensing the light. The most obvious example of this is the Turkish painter Eşref Armağan. Although blind from birth, he can draw pictures of things very successfully. His paintings are consistent with reality, and his drawings are highly artistic [14]. The relationship between the eye and brain explains human vision. It is also accepted that there is a visual brain in human structure [15]. However, living things without a brain can also fulfill the functions of vision. Taking an MR image of a person who has undergone a hemispherectomy will present an interesting result. According to a study on six hemispherectomized individuals, the two hemispheres made their own networks (including functions such as speech, walking) in a single hemisphere. Accordingly, our thinking, reasoning, processing, and integration of all information, interpretation is not dependent on the whole brain; instead, they depend on consciousness. The brain is only a material that activates the functions.

Thus, in this case, if both sides of a human brain are taken, thinking, reasoning and processing of all information will be realized again. In other words, even if there is no organ, light functions at a certain level. It is the light that allows seeing in normal living things. The eye is the window of the body. The window is an organ. Since light provides the main task of seeing, even if the eye window is closed, vision and other actions may occur again. The forthcoming example is noteworthy to realize that seeing is not directly related to the brain. In this case, a man lost $90 \%$ of his brain as a result of discomfort when he was a baby but is now married and has two children. With an IQ of 75, the man had no mental disability. The scientists are looking for an explanation to understand how a man who lost $90 \%$ of his neurons can still live a healthy life [16]. Not all living things have a brain. However, they can carry out brainlike operations. Although plants do not have brain neurons like other living things, they have a memory, and they can even count numbers, act in spiritual behaviors, react to people's feelings and thoughts, feel sad or activate positive events against a negative situation. Plants also have an emotional connection with their caregivers. Plants react to the feelings of their owners, even those who are miles away. Plants can perceive and respond in parallel with their emotions [17]. There are also beings with no brain [18]. For example, the jellyfish (Jellyfish). Jellyfish have no brain [19]. They have no heart. However, it has a very basic nerve group at the base of their tentacles. With these nerves, they are able to touch, perceive temperature, salinity, and so on. Jellyfish also are able to communicate through their nerve endings even though they do not have brains. It has neural networks, which is sensitive to light. This is similar to neurons in humans, which are activated by contact with light.

There is also the example of the organism called Blob, which has no brain, but has consciousness and perception. When two Blobs were combined, the knowledgeable Blob passed on to the other. Blob can digest food even though it has no mouth and stomach. At the same time, when divided by half, Blob can recover quickly [20]. Humans have receptors that provide the senses. 
These receptors are activated by light. These receptors, through light, make the human body functional. Actually, people can think and move without a brain. Indeed, there are optogenetics that use light to control the activity of genes. Fussenegger and colleagues created a small, implantable cartridge containing human stem cells designed to produce a protein called alkaline phosphatase (SEAP), which is secreted when cells are exposed to near-infrared light. The researchers then put this cartridge under a mouse with a LightEmitting Diode (LED) near-infrared. The light caused the implanted cells to begin producing SEAP that passed through the cartridge and into the bloodstream of the mouse [21].

Rapidly adapted receptors are most suitable for transmitting signals related to sudden changes in the environment or vibrational fluctuations. The tension-sensitive muscle shuttle is a good example of a tonic receptor for adaptation. The receptors that exist to see are found in the eye. The conversion of light into a mental image is achieved through receptors because the eye has an average of $70 \%$ of all receptors in the body [22]. Living things have similar eyes, but they do not necessarily see in the same way. Indeed, humans, cats, dogs, horses, and ruminants all differ in what they see. Although they look at the same thing, they see the world differently. Dogs and cats can see better at night than human beings, as their light receptors are specialized for low-light vision [23]. This again underlines the fact that there is no direct relationship between vision and the eye. Consciousness also can activate brain movements without a visual function. Although the eyelids are closed while people are asleep, the eyes move when dreaming [24].

Meanwhile, because of blindness, unusual activities have been observed in areas where no activity in the brain should be performed as the blind cannot see. As a matter of fact, a blind person can read the Braille Alphabet with their hands. When monitored via MR imaging while reading, the visual center of the brain was activated during such reading, instead of the part that typically controls the fingers [25]. As the visual part of the brain can perceive the light and act, consciousness can activate the visual brain movements even though the eye does not see. This happens without eyes and seeing. The visual perception of the human brain is a consciousness that occurs in itself, not the eye: this consciousness, namely light thinking, involves reasoning, processing, integration, and interpretation of all information.

\section{Conclusion}

Light is the most important factor in the first formation of the universe. The relationship between matter and light is the main factor in the formation of matter, as in the first formation. The functionality of the human body and organs is also realized by light. Light is also effective in the treatment of diseases. According to quantum theory, the transfer of matter is possible. It can be divided and divided. Light also has the ability to divide and be located in more than one place. There is light at the core of the matter. The more transparent the matter, the more it becomes a derivative of light, and the more light that has its properties. Tesla said that this could happen with consciousness. To me, it is the light that makes up the consciousness that Tesla speaks of. Everything in the universe comes to life with light, and thanks to the light, it exists and continues to exist. Therefore, it is more accurate to focus on the connection of the brain with light, rather than directly linking consciousness to the brain. Indeed, according to Herbert Spencer, the brain involves a limited number of fibers, while cells become the seat of a relatively unlimited number of perceptions [26].

In other words, although consciousness seems to be happening in the brain, the real source of consciousness is not the brain, but light. Therefore, the brain is not essential for consciousness. The existence of living things that do not have a brain or just half confirms this thesis. Consciousness thought occur in the brain, though not necessarily with the brain, but with light. Because consciousness is light, light also has its own consciousness. Since light is infinite, it continues its activity in the whole world of being. Every human cell has a relationship with light. Each cell has its own frequency of light. For example, some sterols change to calciferol (vitamin D1) with ultraviolet effect. Vitamin D1 is not pure. The pure form of this is vitamin D2. A provitamin (7-dehydrocholesterol), which is found in human skin, is also converted to a vitamin by radiation. This is called vitamin D3. Considering that radiation is a kind of light, the effect of light on the cell can be further understood [27]. Of course, the eye can see through light. However, some living things, such as cats and dogs, see better in low light. In addition, people like the Turkish painter Armağan, who is blind from birth, successfully possess many of the phenomena gained by seeing without eye. Accordingly, vision, not the eye, is formed by light. Mr. Armağan perceives objects by touching them with his hand. Jellyfish, which have no brain, also perceive objects with tentacles and creates consciousness. In all these examples, the main factor is light.

Every organ and every functioning of the human body has one hour, and all beings in the universe have a certain hour. All these timings are based on the effect of light since light is the source of life in the universe and the world. Moreover, the intensity of light varies according to the days and times and also to specific places. At the same time, light changes according to night and day. Therefore, the effect of light varies according to the conditions in question. Accordingly, there are two kinds of light, one for the night and the other for the day, on a 24-hour day. The light of the night is dark light. This dark light is part of the cosmic dark light that covers the entire universe. There is light beyond our perceptions that exist in the universe. One kind of this light is Cosmic Dark light. Darkness is the true face of light. In more straightforward terms, darkness is the source of light in the universe. As Jesus claims: "The people walking in darkness have seen a great light; on those living in the land of the shadow of death, a light has dawned" [28]. Some objects feed directly from dark light. Indeed, the pineal gland becomes functional in the absence of light, that is, in the dark. Thus, the source activation for the pineal gland is again this dark light. 
There are beings in the universe that are not visible to humans. Their reasons for not appearing are dark. With the manifestation of light, rays/light from the sun, moon, and stars are projected to the air in the atmosphere, and are projected to the aether out of the atmosphere; thus colorless objects appear in color and exist. Aether is also active due to light outside the atmosphere [29]. Because the ether matter is a dark light. According to a category made in connection with light, objects are either lighted as stars or lightless as captives. Contrary to what is believed, [30] black holes are not lightless, they have cosmic dark light, and it is the gateway to another dimension of being of the universe. Light is infinite. But it cannot be divided forever. It can be divided up to a certain form. If it continues to divide further, the form of the light breaks down and other forms emerge. There are also varieties of light and light layer by layer. Therefore, the universe is layer by layer. The light on the earth is different. Many things can be done on the light that we have on earth. The intensity of the light can be increased. The intensity of light depends on time and space, the position of the moon, the sun and the planets, and depending on the chemical substance, that increase the intensity of light. The most intense period of light in the world is the time before the sun rises and sets.

The light in the realm of matter is different. Many things can be done on the light in the realm of matter. With light, a substance can be transported very quickly from one place to another. Light can also be used to travel to the past. After the light of the substance realm is finished, a different kind of light will be encountered because the light is divided into a species in itself. Light can be transported very quickly from one place to another. With light, a substance can be transported very quickly from one place to another. Once the light of the material world is finished, a different kind of light will emerge. Human perceptions change according to the intensity of the light.

\section{References}

1. Kusakabe M, Kajino T, Yoshida T, Mathews GJ (2010) Signature of longlived relic particles on primordial light element abundances in Big Bang nucleosynthesis. The $10^{\text {th }}$ International Symposium on Origin of Matter and Evolution of Galaxies Omeg 2010 AIP Conference Proceedings 1269: 63-68.

2. Devlin H (2018) Cosmic dawn: astronomers detect signals from first stars in the universe. The Guardian.

3. The Holy Bible Genesis 1-4 The Holy Bible John 1:1-5; Qur'an 24:35, 40; 39:69; 81:1-2.

4. Plato (2017) Republic. Istanbul Is Bank Publishing 508.

5. Hu W, White M (2004) The Cosmic symphony. Scientific American 290: 44-53.

6. Rugg BK, Krzyaniak MD, Phelan BT, Ratner MA, Young RM, et al. (2019) Photodriven quantum teleportation of an electron spin state in a covalent donor-acceptor-radical system. Natural Chemistry 11(11): 981-986.

7. Pirandola S, Eisert J, Weedbrook C, Furusawa C, Braunstein SL (2015) Advances in quantum teleportation. Nature Photonics 9: 641-652.
8. Wang J, Sciarrino F, Laing A, Mark G Thompson (2019) Integrated photonic quantum technologies. Nature Photonics.

9. Ayuso D, Neufeld O, Ordonez AF, Decleva P, Lerner G, et al. (2019) Synthetic chiral light for efficient control of chiral light-matter interaction. Nature Photonics 13: 866-871.

10.Sophia TC Leung, R Anne McKinney, Alanna J Watt (2019) Brain Development: The impact of light during the night. eLife.

11. Figueiro MG (2017) Disruption of Circadian Rhythms by Light During Day and Night. Curr Sleep Med Rep 3(2): 76-84.

12. Nash TR, Chow ES, Law AD, Fu SD, Fuszara E, et al. (2019) Daily blue light exposure shortens lifespan and causes brain neurodegeneration in Drosophila. Nature Partner Journal.

13. Reid, Dunn K, Young RJ, Amu J, Donovan T, et al. (2017) The Human fetus preferentially engages with face-like visual stimuli. Current Biology 27(12):1825-1828.

14. Hayhoe SJ, Cohen R, Carrizosa, Helena Garcia (2019) Locke and Hume's philosophical theory of color is investigated through a case study of Esref Armagan, an artist born blind. Journal of Blindness Innovation Research 9(1).

15. Milner ADG, Melvyn A (1998) The Visual brain in action. Psyche 4(12): 1-8.

16. Feuillet L, Dufour H, Pelletier J (2007) Brain of a white-collar worker. Lancet 370(9583): 262.

17. Gagliano M, Renton M, Depczynski M, Mancuso S (2014) Experience teaches plants to learn faster and forget slower in environments where it matters. Oecologia 175(1): 63-72.

18. Herrera R C, Pai VP, Moran KM, Lemire JM1, Levin M (2017) The brain is required for normal muscle and nerve patterning during early Xenopus development. Nat Commun 8(1): 587.

19. Rüdiger W (2005) Brainless eyes. Nature. 435: 157-159.

20. Wetsman N (2017) These jellyfish don't have brains, but still somehow seem to sleep. Popular Sciences.

21. Mcrae M (2019) Here's the Truth behind The Blob'-Paris Zoo's latest display that's gone viral. Science Alert.

22. Reardon S (2014) Human brainwaves light up mouse genes. Nature.

23. McDougal DH, Gamlin PD (2015) Autonomic Control of the Eye. Compr Physiol 5(1): 439-473.

24. Bernstein MH, Pease DC (1959) Electron Microscopy of the Tapetum Lucidum of the Cat. J Biophy Biochem Cytology 5(1): 35-39.

25. AndrillonT, Nir Y, Cirelli C, Tononi G, Fried I (2015) Single-neuron activity and eye movements during human REM sleep and awake vision. Nat Commun 6: 7884.

26. Bola L, Siuda KK, Paplińska M, Ewa Sumera, Maria Zimmermann, et al. (2017) Structural reorganization of the early visual cortex following Braille training in sighted adults. Sci Rep 7: 17448.

27. Smith CUM (1892) Evolution and the Problem of Mind: Part I. Herbert Spencer. Jo History Biol 15(1): 55-88.

28. Shanaz AG, Shuryak I, Morton SR, Amundson SA, Brenner DJ (2019) New approaches for quantitative reconstruction of radiation dose in human blood cells. Nature Sci Rep 9: 18441.

29. Whittaker ET (1951) A history of the theories of aether and electricity: The classical theories. Nelson.

30. Camille MC (2019) Sky \& Telescope. 
ISSN: 2574-1241

DOI: $10.26717 / B J S T R .2020 .25 .004224$

Alemdar E. Biomed J Sci \& Tech Res

(c) (P) This work is licensed under Creative BY Commons Attribution 4.0 License

Submission Link: https://biomedres.us/submit-manuscript.php

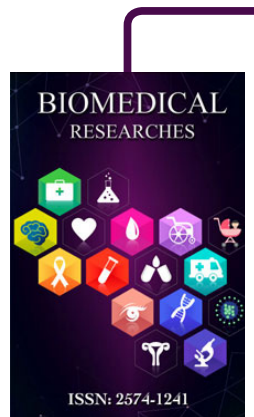

Assets of Publishing with us

- Global archiving of articles

- Immediate, unrestricted online access

- Rigorous Peer Review Process

- Authors Retain Copyrights

- Unique DOI for all articles

https://biomedres.us/ 